\title{
Towards Robust Task Execution for Domestic Service Robots
}

\author{
A. Kuestenmacher, N. Akhtar, P. G. Plöger \\ Bonn-Rhein-Sieg University of Applied \\ Sciences, Grantham-Allee 20, 53757 \\ Sankt Augustin, Germany
}

\author{
G. Lakemeyer \\ RWTH Aachen University, \\ Templergraben 55, 52056 \\ Aachen, Germany
}

\begin{abstract}
In the field of domestic service robots, recovery from faults is crucial to promote user acceptance. In this context we focus in particular on some specific faults, which arise from the interaction of a robot with its real world environment.

Even a well-modelled robot may fail to perform its tasks successfully due to unexpected situations, which occur while interacting. These situations occur as deviations of properties of the objects (manipulated by the robot) from their expected values. Hence, they are experienced by the robot as external faults.

In this paper we present two approaches to handle external faults which result from inadequate descriptions of a planner operator. In both approaches we assume that the robot is able to detect the occurrence of the fault at the planning level by monitoring the effects of an executed action. In our work we limit the scope of the sources of external faults to natural physical phenomena. Hence, we do not consider cases in which an external agent (e.g. another robot, a human being) is the cause of a detected fault. We apply the proposed approaches to scenarios in which the robot performs a manipulation task (pick and place).
\end{abstract}

\section{Introduction}

This paper is an extended abstract of the journal article Towards Robust Task Execution for Domestic Service Robots published in Journal of Intelligent and Robotic Systems 2013 (Kuestenmacher et al. 2013).

Fault diagnosis and tolerance is considered a major challenge in the robotics community. Many contributions have been made in the detection and diagnosis of the faults which occur due to failure or malfunctioning of robot's internal components (e.g. sensors and actuators etc.). However, to achieve true robustness in robotics, it is not sufficient to just successfully diagnose the faults that occur within a robot's internal components. A robot can only achieve true robustness if it also has the ability to handle unforeseen situations which may result in a failure to complete its actions. For instance, consider the following scenarios.

Copyright (C) 2014, Association for the Advancement of Artificial Intelligence (www.aaai.org). All rights reserved.
Scenario1: A robot is required to place a die on a planar surface above the ground (i.e. a table). We assume all internal components of the robot function perfectly. However, after the completion of the robot's action, the die falls on the floor instead of remaining on the table. The reason for this unsuccessful completion of the robot's action is simple: After its release, the die lands on the table on its edge. This makes the die roll and fall on the floor.

Scenario 2: The robot is picking up a bottle from the table and the surface of this bottle is slippery. When the robot picks up the bottle, the bottle slips from the robot's gripper and falls on the table. Once again the robot does not detect any fault in its internal components. Still it is not able to complete its action successfully.

In our work we group the scenarios described above into a class called inadequate description of planner operator. For example, in scenario 1 the main reason for unsuccessful completion of the releasing action is the gripper holding the die in the wrong way. The wrong grip (just before the release) is not rectified by the robot before executing the action because the current state of the die fulfils the inadequately defined preconditions of the release action.

Even humans cannot always guarantee to perform their actions in the desired manner. The reason is the lack of knowledge about possible outcomes of unknown conditions. For humans it is easy to learn how to avoid these external faults in the future, by updating our knowledge through learning from the current unexpected situation by experimentation. In the next sections we will describe two approaches which are applicable to deal with unexpected situations due to inadequate description of planner operators.

Our first approach illustrates how naïve physics knowledge can be formalized such that reasoning about external faults can benefit from it. The second approach uses simulation as an oracle to avoid external faults that occur during a releasing action.

\section{Naïve Physics Approach}

The situations mentioned above are only two among countless possibilities which can result in external faults for the actions of picking and placing an object. It is not possible to model all such situations in advance. Furthermore, the use of such models for diagnosis purposes can be computationally 
very expensive.

On the other hand, we can see that human beings also face such situations in their daily life. Normally, we do not use any computational model or even any quantitative information to handle these situations. Humans use commonsense to avoid such faults in the future. Therefore, we propose to use naive physics knowledge for reasoning about external faults in robotics.

In his work (Hayes 1979) Hayes proposes to formalize everyday knowledge of the physical world in a declarative symbolic theory. In principle, such a theory represents the naive physics knowledge which can be used for commonsense reasoning about everyday physical phenomena. This proposal of Hayes is one of the widely acclaimed ideas in AI, but it has never been truly followed (Davis 1998). For commonsense reasoning, the literature in AI is mostly concerned with what is more appropriately called qualitative physics (de Kleer and Brown 1990). In contrast to Hayes' proposal of naive physics, qualitative physics concentrates on reasoning programs rather than knowledge representation. Furthermore, qualitative physics emphasizes on restricted systems rather than daily life physical phenomena.

One approach of representing commonsense physical knowledge, which is based on Hayes' notion of naive physics, is the methodology of microworlds (Davis 1998). Davis proposes to structure the knowledge in microworlds, where "a microworld is an abstraction of a small part of physical interactions, sufficient to support some interesting collection of inferences". Some examples of microworlds are component-based electronics (de Kleer and Brown 1990), rigid object kinematics and rigid objects dynamics. Structuring the knowledge in chunks makes its formalization easier. Compared to Hayes' proposal, any knowledge formalized using the microworlds approach is more inferencing oriented. This also implies that by keeping in view the collection of inferences to be supported by a microworld, we can limit the scope of the knowledge to be formalized for that microworld. In our opinion, this aspect of the microworlds approach makes it more practical in comparison to the original proposal of Hayes.

Reasoning about the unknown external faults in robotics requires the ability to reason about physical phenomena encountered in our daily life. Approaches in qualitative physics (QSIM (Kuipers 2001) and qualitative process theory (Forbus 1984) etc.) are inadequate for such reasoning in general (Davis 1988).

On the other hand it is also true that in current AI literature there does not exist any large scale formalization of daily life physical phenomenon (formalized in a naive physics theory or structured in microworlds) which can be used directly for unknown external faults in robotics. Therefore, in the work proposed here, we use insights from the works referenced above to formalize a small body of naive physics knowledge for reasoning about unknown external faults in robotics. Our approach illustrates how naive physics knowledge can be formalized that reasoning about unknown external faults can benefit from it.

We will therefore use naïve physics concepts to find infor- mation about the cause of the fault. This information is produced in the form of hypotheses. The naïve physics concepts which are represented by the physical properties of objects, are formalised in a logical framework. To reason about the fault, we apply a qualitative version of physical laws to these properties. Since effective reasoning about any external fault requires information about relevant properties and physical laws, we associate different properties and laws to different types of faults. The underlying ontology of this association is based on studies conducted (by other researchers) on reasoning of physics novices about everyday physical phenomena. By interpreting the results of the reasoning process, the robot deduces the hypotheses about the situations that could have caused the detected faults. The details of the described approach is presented in our original article (Kuestenmacher et al. 2013)

We apply our scheme to the aforementioned scenario in which the die falls on the floor instead of staying on the table. As the result the approach generates two separate lists of hypotheses for the phenomena of gravity and air. The hypotheses shown bellow are stated in Prolog syntax.

For gravity following hypotheses are generated for the given scenario.

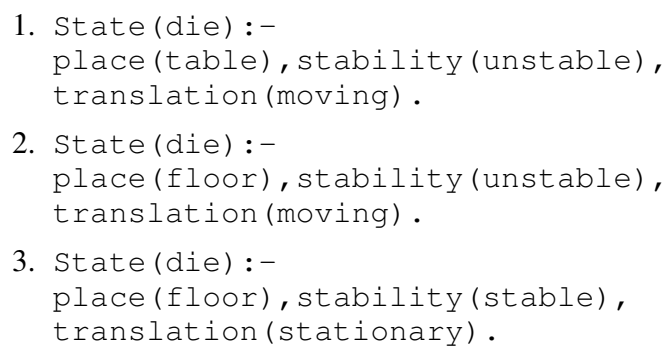

For a ir, the naïve physic approach generates following hypotheses.

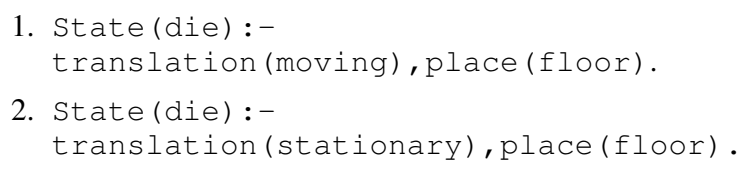

As stated earlier, each of the generated hypotheses represents a situation that can be the cause of the detected fault. As human beings, we can immediately see that hypothesis 1 for gravity, is the one with the highest probability for the considered scenario. Interpretation of this hypothesis gives the reason of the detected fault.

We also apply our approach to the other scenario in which a robot is simply picking up a bottle. When the robot picks it up, the bottle slips from the gripper of the robot and falls on the table. Again, the detected fault is categorized under the type location. Therefore, the approach also generates two lists of hypotheses for gravity and air phenomena.

\section{Simulation-Based Approach}

In our running example, the naïve physics approach was able to produce the information that the die has fallen on the 
floor because its center of gravity had left the top of the table (because of any intrinsic property of the die) after its release. This information can be used in modifying the preconditions of the relevant planning operator of the robot such that they also incorporate the observed anomalous behavior of the object.

This is done in order to show that meaningful information about the occurred situation can be extracted from naive physics knowledge using the proposed methodology.

Despite of successful application results, the pure symbolic, abstract approach is not informative enough to reason about physical properties of the object. How can we conclude from the faulty symbolic predicate on (table, die) that the die was released with incorrect orientation which is probably the true cause of the fault?

On the other hand simulation-based approaches provide us with more accurate low-level details about the behaviour of an object which is required to reason about external faults.

In our second approach we present a technique which utilizes a physics-based simulator in order to suggest an optimal solution for external faults in an action during the release. The idea of using a physical simulator as a tool which represents a realistic execution of an action in real world environment is not new. The current researches in robotics (which are detailed in the original article (Kuestenmacher et al. 2013)) use simulation for navigation, motion planning and reasoning about task execution.

Therefore we call our second technique simulation-based approach (SBA).

SBA is presented as a four-step scheme that requires the following inputs: a simulated example of the expected behaviour of the object and a definition of the planning operator for the action that results in a detected fault. The approach first finds a symbolic description of the simulated behaviour of the object. This description is presented as logical expressions, which consist of conjunctions of basic atomic facts. Each atomic fact represents a different aspect of a given state of the objects in the simulation. We refer to the atomic facts collectively as the description vocabulary. The description vocabulary is derived from the concepts in the area of qualitative spatial representation. SBA creates different examples of object behaviour (in simulation) and uses the symbolic description to autonomously label these examples as desired or undesired. These labelled examples are then utilised by a learning algorithm to find the best initial state of the object. Performing the action in this initial state avoids the occurrence of external faults. Once the learning algorithm is exposed to the labelled examples, it can also be used for predicting the desirability of an initial state of the object. We use these results to modify the preconditions of the action of the robot. More detail information is given in our journal article (Kuestenmacher et al. 2013)

We performed different experiments of the action of dropping objects on various surfaces using SBA in a simulation environment. For example in one experiment the example simulation (expected by the approach as an input) shows that a die is dropped on a table. During the other experiment the example simulation shows that a carton is dropped over the centre of an empty table such that it stands tall on the table. However, before generating the training instances we update this model by placing different solid objects on the table. In this experiment the approach was able to find the correct results despite outdated models of the objects in the example simulation because it was able to correctly label the training instances. The correct labeling of the training instances, in turn, is made possible by the fact that the approach uses only qualitative information in the simulation description of the example simulation. This information remains unaffected by the quantitative changes caused by the updates in the models of the objects. Hence, SBA is able to find the correct results. In the last experiment the example simulation shows that a ball is thrown towards an empty basket, such that it falls and stay inside the basket. Similar to the case of previous experiment, we update the model of the basket in this experiment. The approach generates the instances of throwing the ball, using this updated model which contains different solid objects inside the basket.

Results of these experiments clearly showed that with its help a robot can not only avoid external faults by selecting a safe releasing state for the object but can also predict desirability of a given releasing state of the object with considerable accuracy.

Broadly speaking, the main idea behind the proposed approach for avoiding external faults can be summarised as give a robot an example of the action to be executed and let it find the safest way to do it.

\section{Discussion}

The implementation of the naive physics approach is limited to a particular (type of) robot. That is, the developed theories, ontologies and physical laws are developed for a particular robot and can be exported (with minimum changes) only to the robots with similar capabilities. Therefore, to use this approach for external fault reasoning in a real robot, we first need to understand the capabilities (i.e. performable tasks) of the robot. To this end, one needs to understand the knowledge of planning operators and methods used in the robot. Based on this understanding, the types of faults must be categorised and extended.

Then the ontology of the properties has to be developed. It should be noted that there is no correct or wrong ontology for properties. The ontology used in this work is derived form the substance schema. It is expected that this ontology can vary greatly if the types of faults are different. For each type of fault, a pool of definitions (i.e. framework) is to be developed. The development of definitions can follow the approach outlined in this work. However, it may be possible to utilize definitions more effectively by letting a knowledge base know objects and their intrinsic properties. For example, if the knowledge base already stores the names of fixed objects in its environment then there is no need to define movable, fixed etc.. Limitations of this approach are like the other qualitative reasoning techniques (Mösenlechner and Beetz 2011). They are too abstract to 
represent geometric and physic properties of an object in its environment for realistic reasoning.

In our future research we will implement the simulationbased mechanism to our service robot Jenny. Jenny is a CareO-botIII system equipped with a 7 DOF light-weight KUKA arm and a 7-DOF Schunk Dexterous-Hand, an omnidirectional platform and many high end sensors (Reiser et al. 2009). Jenny will have to perform everyday manipulation activities such as pick-and-place tasks in a dynamic domestic environment. For the successful implementation we have to make sure that the following assumptions about the robot and the situations hold true:

- The robot is equipped with planning capabilities representing the state of the world as a conjunction of logical predicates. In order to handle external faults their occurrence has to be detected. Therefore, the robot has to be able to monitor its behaviour continuously while executing the generated plan. During the monitoring phase the symbolic state of the system (robot and its environment) is compared to the current observable state of the world. In case of inconsistencies the last executed action is marked as faulty. This action will then be used as an input to our approach. The other input parameter is an example simulation of the marked action. The example simulation also includes a domestic environment that has to be updated accordingly to the changes in the robot's current environment (for example a table which was previously known to be empty may have some objects on it at the time of execution).

- There is no involvement of external agents in the occurrence of faults (i.e. all faults are caused by the robot itself).

The SBA is too time consuming and may work only offline. To overcome this problem we propose to run many simulated experiments in parallel on a PC with 48 cores. Therefore we suggest to set up the approach on the external multiprocessor PC outside the robot. The robot may then send necessary input data to this external PC, which will start the simulation-based approach and then send the solution back to the robot.

Our first results using the simulation based approach were successful. They encourage further exploration and extension of this work to other robotic actions (e.g. picking objects) and environments. Another important future direction for us is to use labelled instances of simulated behaviour of a given action to learn a general solution for it. These solutions may suggest an optimal way to perform particular actions successfully not only with the current object but also with any scaled objects of similar geometrical shape.

\section{Acknowledgements}

This work was sponsored by the B-IT foundation and the Strukturfond des Landes Nordrhein-Westfalen for female PhD students.

\section{References}

Davis, E. 1988. A logical framework for commonsense predictions of solid object behaviour. Artificial Intelligence in Engineering 3(3): 125 - 140.

Davis, E. 1998. The naive physics perplex. AI Magazine 19:51-79.

de Kleer, J., and Brown, J. 1990. A qualitative physics based on confluences. San Francisco, CA, USA: Morgan Kaufmann Publishers Inc. 83-126.

Forbus, K. 1984. Qualitative process theory. Artificial Intelligence. 24:85-168.

Hayes, P. 1979. The naive physics manifesto. In Michie, D., ed., Expert Systems in the Micro Electronic Age. Edinburgh University Press. 242-270.

Kuestenmacher, A.; Akhtar, N.; Plöger, P.; and Lakemeyer, G. 2013. Towards robust task execution for domestic service robots. Journal of Intelligent and Robotic Systems.

Kuipers, B. 2001. Qualitative simulation. Artificial Intelligence 29:289-338.

Mösenlechner, L., and Beetz, M. 2011. Parameterizing actions to have the appropriate effects. In IEEE/RSJ International Conference on Intelligent Robots and Systems (IROS), 4141-4147.

Reiser, U.; Connette, C.; Fischer, J.; Kubacki, J.; Bubeck, A.; Weisshardt, F.; Jacobs, T.; Parlitz, C.; Hägele, M.; and Verl, A. 2009. Care-O-bot $\AA 3$ - Creating a product vision for service robot applications by integrating design and technology. In IEEE/RSJ International Conference on Intelligent Robots and Systems (IROS), 1992-1998. 